\title{
Pointwise observation of the state given by parabolic system with boundary condition involving multiple time delays
}

\author{
ADAM KOWALEWSKI
}

\begin{abstract}
Various optimization problems for linear parabolic systems with multiple constant time delays are considered. In this paper, we consider an optimal distributed control problem for a linear parabolic system in which multiple constant time delays appear in the Neumann boundary condition. Sufficient conditions for the existence of a unique solution of the parabolic equation with the Neumann boundary condition involving multiple time delays are proved. The time horizon $T$ is fixed. Making use of the Lions scheme [13], necessary and sufficient conditions of optimality for the Neumann problem with the quadratic cost function with pointwise observation of the state and constrained control are derived.
\end{abstract}

Key words: distributed control, parabolic system, time delays, pointwise observation.

\section{Introduction}

Infinite dimensional distributed parameter systems with delays can be used to describe many phenomena in the real world. As is well known, heat conduction, properties of elastic-plastic material, fluid dynamics, diffusion-reaction processes, transmission of the signals at the certain distance by using electric long lines, etc., all lie within this area. The object that we are studying (temperature, displacement, concentration, velocity, etc.) is usually referred to as the state.

We are interested in the case where the state satisfies proper differential equations that are derived from basic physical laws, such as Newton's law, Fourier's law etc. The space in which the state exists is called the state space, and the equation that the state satisfies is called the state equation. Consequently, by an infnite dimensional system we mean one whose corresponding state space is infinite dimensional. In particular, we are interested in the cases where the state equations are one of the following types: partial differential equations, integro-differential equations, or abstract evolution equations.

The Author is with AGH University of Science and Technology, Institute of Automatics and Biomedical Engineering, 30-059 Cracow, al. Mickiewicza 30, Poland, e-mail: ako@agh.edu.pl.

The research presented here was carried out within the research programme AGH University of Science and Technology, No. 11.11.120.396.

Received 25.01.2016. 
Very important optimization problems associated with the optimal control of distributed parabolic systems with time delays appearing in the boundary conditions have been studied recently in Refs. [1] - [12] and [15], [16].

In this paper, we consider an optimal distributed control problem for a linear parabolic system in which multiple constant time delays appear in the Neumann boundary condition.

Such systems constitute in a linear approximation, a universal mathematical model for the plasma control process [12].

Sufficient conditions for the existence of a unique solution of such parabolic equations with the Neumann boundary conditions involving multiple time delays are proved.

In this paper, we restrict our considerations to the case of the distributed control for the Neumann problem. Consequently, we formulate the following optimal control problem. We assume that the cost function has the quadratic form with pointwise observation of the state. Moreover, the time horizon is fixed in our optimization problem. Finally, we impose some constraints on the distributed control. Making use of the Lions framework [13] necessary and sufficient conditions of optimality with the quadratic cost function with pointwise observation of the state and constrained control are derived for the Neumann problem.

\section{Existence and uniqueness of solutions}

Consider now the distributed-parameter system described by the following parabolic delay equation

$$
\begin{gathered}
\frac{\partial y}{\partial t}+A(t) y=v \quad x \in \Omega, t \in(0, T) \\
y(x, 0)=y_{0}(x) \quad x \in \Omega \\
\frac{\partial y}{\partial \eta_{A}}=\sum_{i=1}^{m} y\left(x, t-h_{i}\right)+u \quad x \in \Gamma, t \in(0, T) \\
y\left(x, t^{\prime}\right)=\Psi_{0}\left(x, t^{\prime}\right) \quad x \in \Gamma, t^{\prime} \in\left[-h_{m}, 0\right)
\end{gathered}
$$

where: $\Omega \subset R^{n}$ is a bounded, open set with boundary $\Gamma$, which is a $C^{\infty}$ - manifold of dimension $n-1$. Locally, $\Omega$ is totally on one side of $\Gamma \cdot \frac{\partial y}{\partial \eta_{A}}$ is a normal derivative at $\Gamma$, directed towards the exterior of $\Omega$,

$$
\begin{array}{cc}
y \equiv y(x, t ; v), & v \equiv v(x, t), \\
Q=\Omega \times(0, T), & \bar{Q}=\bar{\Omega} \times[0, T], \\
\Sigma=\Gamma \times(0, T), & \Sigma_{0}=\Gamma \times\left[-h_{m}, 0\right)
\end{array}
$$


$h_{i}$ are specified positive numbers representing time delays such that $0 \leqslant h_{1}<h_{2}<\ldots<$ $h_{m}$ for $i=1, \ldots, m, \Psi_{0}$ is a initial function defined on $\Sigma_{0}$.

The operator $A(t)$ has the form

$$
A(t) y=-\sum_{i, j=1}^{n} \frac{\partial}{\partial x_{i}}\left(a_{i j}(x, t) \frac{\partial y(x, t)}{\partial x_{j}}\right)
$$

and the functions $a_{i j}(x, t)$ satisfy the condition

$$
\begin{array}{r}
\sum_{i, j=1}^{n} a_{i j}(x, t) \Phi_{i} \Phi_{j} \geqslant \alpha \sum_{i=1}^{n} \Phi_{i}^{2} \quad \alpha>0, \\
\forall(x, t) \in \bar{Q}, \forall \Phi_{i} \in R
\end{array}
$$

where: $a_{i j}(x, t)$ are real $C^{\infty}$ functions defined on $\bar{Q}$ (closure of $Q$ ). The equations (1) - (4) constitute a Neumann problem.

First we shall prove sufficient conditions for the existence of a unique solution of the mixed initial-boundary value problem (1) - (4) for the case where $v \in L^{2}(Q)$.

For this purpose, for any pair of real numbers $r, s \geqslant 0$, we introduce the Sobolev space $H^{r, s}(Q)$ ( [14], Vol. 2, p.6) defined by

$$
H^{r, s}(Q)=H^{0}\left(0, T ; H^{r}(\Omega)\right) \cap H^{s}\left(0, T ; H^{0}(\Omega)\right)
$$

which is a Hilbert space normed by

$$
\left(\int_{0}^{T}\|y(t)\|_{H^{r}(\Omega)}^{2} d t+\|y\|_{H^{s}\left(0, T ; H^{0}(\Omega)\right)}^{2}\right)^{\frac{1}{2}}
$$

where: the spaces $H^{r}(\Omega)$ and $H^{s}\left(0, T ; H^{0}(\Omega)\right)$ are defined in Chapter 1 ( [14], Vol.1) respectively. Consequently, some properties and central theorems for the functions $y \in$ $H^{r, s}(Q)$ are given in [8], [11] and [14].

The existence of a unique solution for the mixed initial-boundary value problem (1) - (4) on the cylinder $Q$ can be proved using a constructive method, i.e., first, solving (1) - (4) on the subcylinder $Q_{1}$ and in turn on $Q_{2}$, etc. until the procedure covers the whole cylinder $Q$. In this way the solution in the previous step determines the next one. For simplicity, we introduce the following notations:

$$
\begin{gathered}
E_{j} \triangleq((j-1) \lambda, j \lambda) \text { where } \lambda=\min h_{i}, \quad Q_{j}=\Omega \times E_{j}, \\
\Sigma_{j}=\Gamma \times E_{j} \text { for } j=1, \ldots K .
\end{gathered}
$$

Using the results of Section 14 ([13], pp. 182-185) we can prove the following result. 
Lemma 1 Let

$$
\begin{gathered}
v \in L^{2}(Q) \\
y_{j-1}(\cdot,(j-1) \lambda) \in L^{2}(\Omega) \\
q_{j} \in H^{1 / 2,1 / 4}\left(\Sigma_{j}\right)
\end{gathered}
$$

where:

$$
q_{j}(x, t)=\sum_{i=1}^{m} y_{j-1}\left(x, t-h_{i}\right)+u(x, t)
$$

Then, there exists a unique solution $y_{j} \in H^{2,1}\left(Q_{j}\right)$ for the mixed initial-boundary value problem (1), (3), (10).

Proof We observe that for $j=1$,

$$
\left.\sum_{i=1}^{m} y_{j-1}\right|_{\Sigma_{0}}\left(x, t-h_{i}\right)=\sum_{i=1}^{m} \Psi_{0}\left(x, t-h_{i}\right) .
$$

Then the assumptions (10) and (11) are fulfilled if we assume that $\Psi_{0} \in H^{1 / 2,1 / 4}\left(\Sigma_{0}\right)$, $u \in H^{1 / 2,1 / 4}(\Sigma)$ and $y_{0} \in L^{2}(\Omega)$. These assumptions are sufficient to ensure the existence of a unique solution $y_{1} \in H^{2,1}\left(Q_{1}\right)$. Next for $j=2$ we have to verify that $y_{1}(\cdot, \lambda) \in L^{2}(\Omega)$ and $q_{2} \in H^{1 / 2,1 / 4}\left(\Sigma_{2}\right)$. Really, from the Theorem 3.1 ( [14], Vol.1, p.19) we can prove that $y_{1} \in H^{2,1}\left(Q_{1}\right)$ implies that the mapping $t \rightarrow y_{1}(\cdot, t)$ is continuous from $[0, \lambda] \rightarrow$ $H^{1}(\Omega) \subset L^{2}(\Omega)$, hence $y_{1}(\cdot, \lambda) \in L^{2}(\Omega)$. Then using the Trace Theorem ( [14], Vol. 2, p.9) we can verify that $y_{1} \in H^{2,1}\left(Q_{1}\right)$ implies that $\left.y_{1} \rightarrow y_{1}\right|_{\Sigma_{1}}$ is a linear, continuous mapping of $H^{2,1}\left(Q_{1}\right) \rightarrow H^{1 / 2,1 / 4}(\Sigma)$. Assuming that $u \in H^{1 / 2,1 / 4}(\Sigma)$, the condition $q_{2} \in$ $H^{1 / 2,1 / 4}\left(\Sigma_{2}\right)$ is fulfilled.

We shall now summarize the foregoing result for any $Q_{j}, j=3, \ldots, K$.

Theorem 1 Let $y_{0}, \Psi_{0}, u$ and $v$ be given with $y_{0} \in L^{2}(\Omega), \Psi_{0} \in H^{1 / 2,1 / 4}\left(\Sigma_{0}\right)$, $u \in H^{1 / 2,1 / 4}(\Sigma)$ and $v \in L^{2}(Q)$. Then, there exists a unique solution $y \in H^{2,1}(Q)$ for the mixed initial-boundary value problem (1) - (4). Moreover, $y(\cdot, j \lambda) \in L^{2}(\Omega)$ for $j=1, \ldots, K$.

\section{Problem formulation. Optimization theorems}

We shall now formulate the optimal distributed control problem for the Neumann problem. Let us denote by $U=L^{2}(Q)$ the space of controls. The time horizon $T$ is fixed in our problem.

Let $x^{1}, \ldots, x^{\mu}$ be points of $\Omega$. We assume that the observation is $\left\{y\left(x^{j}, t ; v\right)\right\}, 1 \leqslant j \leqslant$ $\mu$ - provided we can attach a meaning to this. 
If we now assume that the coefficients of the operator $A$ in the equation (1) are sufficiently regular, then from the Theorem 1 it follows that

$$
y(v) \in H^{2,1}(Q) .
$$

Hence, $y(v) \in L^{2}\left(0, T ; H^{2}(\Omega)\right)$ and $y\left(x^{j}, t\right)$ has meaning (and " $t \rightarrow y\left(x^{j}, t\right) " \in L^{2}(0, T)$ ) if

$$
H^{2}(\Omega) \subset C^{0}(\Omega)
$$

which is true if (and only if)

$$
\frac{1}{2}-\frac{2}{n}<0 \text { i.e. } n \leqslant 3 .
$$

Hence we make the standing hypothesis that the dimension is $n \leqslant 3$.

Then the observation

$$
C y(v)=\left\{y\left(x^{j}, t ; v\right)\right\} \in\left(L^{2}(0, T)\right)^{\mu} .
$$

The cost function is now given

$$
I(v)=\lambda_{1}\left\|C y(v)-z_{d}\right\|_{\left(L^{2}(0, T)\right)^{\mu}}^{2}+\lambda_{2} \int_{Q}(N v) v d x d t .
$$

If $z_{d}=\left\{z_{d 1}, \ldots, z_{\mu}\right\}$,

$$
I(v)=\lambda_{1} \sum_{j=1}^{\mu} \int_{0}^{T}\left|y\left(x^{j}, t ; v\right)-z_{d j}(t)\right|^{2} d t+\lambda_{2} \int_{Q}(N v) v d x d t
$$

where: $\lambda_{i} \geqslant 0, \lambda_{1}+\lambda_{2}>0 ; z_{d j}(t)$ are given elements in $L^{2}(0, T)$ and $N$ is a positive, linear operator on $L^{2}(Q)$ into $L^{2}(Q)$.

Finally, we assume the following constraint on controls $v \in U_{a d}$, where

$$
U_{a d} \text { is a closed, convex subset of } U
$$

Let $y(x, t ; v)$ denote the solution of the mixed initial-boundary value problem (1)- (4) at $(x, t)$ corresponding to a given control $v \in U_{a d}$. We note from the Theorem 1 that for any $v \in U_{a d}$ the cost function (17) is well-defined since $y(v) \in H^{2,1}(Q)$. The solving of the formulated optimal control problem is equivalent to seeking a $v_{0} \in U_{a d}$ such that $I\left(v_{0}\right) \leqslant I(v) \quad \forall v \in U_{a d}$. Then from the Theorem 1.3 ( [13], p. 10) it follows that for $\lambda_{2}>0$ a unique optimal control $v_{0}$ exists; moreover, $v_{0}$ is characterized by the following condition

$$
I^{\prime}\left(v_{0}\right) \cdot\left(v-v_{0}\right) \geqslant 0 \quad \forall v \in U_{\mathrm{ad}}
$$


Using the form of the cost function given by (17) we can express (19) in the following form

$$
\begin{array}{r}
\lambda_{1} \sum_{j=1}^{\mu} \int_{0}^{T}\left(y\left(x^{j}, t ; v_{0}\right)-z_{d j}(t)\right)\left(y\left(x^{j}, t ; v\right)-y\left(x^{j}, t ; v_{0}\right)\right) d t+ \\
+\lambda_{2} \int_{Q} N v_{0}\left(v-v_{0}\right) d x d t \geqslant 0 \quad \forall v \in U_{a d}
\end{array}
$$

To simplify (20), we introduce the adjoint equation and for every $v \in U_{a d}$, we define the adjoint variable $p=p(v)=p(x, t ; v)$ as the solution of the equation

$$
\begin{gathered}
-\frac{\partial p(v)}{\partial t}+A^{*}(t) p(v)=\lambda_{1} \sum_{j=1}^{\mu}\left(y\left(x^{j}, t ; v\right)-z_{d j}(t)\right) \otimes \delta\left(x-x^{j}\right) \\
x \in \Omega, t \in(0, T) \\
p(x, T ; v)=0 \quad x \in \Omega \\
\frac{\partial p(v)}{\partial \eta_{A^{*}}}(x, t)=\sum_{i=1}^{m} p\left(x, t+h_{i} ; v\right) \quad x \in \Gamma, t \in\left(0, T-h_{m}\right) \\
\frac{\partial p(v)}{\partial \eta_{A^{*}}}(x, t)=0 \quad x \in \Gamma, t \in\left(T-h_{m}, T\right)
\end{gathered}
$$

where

$$
\left\{\begin{array}{l}
g(t) \otimes \delta\left(x-x^{j}\right) \text { is the distribution, } \\
\Psi \rightarrow \int_{0}^{T} g(t) \Psi\left(x^{j}, t\right) d t, \Psi \in \mathcal{D}(Q) \\
A^{*}(t) p=-\sum_{i, j=1}^{n} \frac{\partial}{\partial x_{j}}\left(a_{i j}(x, t) \frac{\partial p}{\partial x_{i}}\right)
\end{array}\right.
$$

The existence of a unique solution for the problem (21) - (24) on the cylinder $Q$ can be proved using a constructive method. It is easy to notice that for given $z_{d}$ and $v$, problem (21) - (24) can be solved backwards in time starting from $t=T$, i.e., first, solving (21) - (24) on the subcylinder $Q_{K}$ and in turn on $Q_{K-1}$, etc. until the procedure covers the whole cylinder $Q$. For this purpose, we may apply Theorem 1 (with an obvious change of variables) to problem (21) - (24) (with reversed sense of time, i.e., $t^{\prime}=T-t$ ).

Lemma 2 Let the hypothesis of Theorem 1 be satisfied. Then, for given $z_{d j}(t) \in L^{2}(0, T)$ and any $v \in L^{2}(Q)$, there exists a unique solution $p(v) \in H^{2,1}(Q)$ for the problem 
(21) - (24) defined by transposition

$$
\begin{gathered}
\int_{Q} p\left(v_{0}\right)\left(\frac{\partial \Psi}{\partial t}+A \Psi\right) d x d t= \\
=\sum_{j=1}^{\mu} \int_{0}^{T}\left(y\left(x^{j}, t ; v_{0}\right)-z_{d j}(t)\right) \Psi\left(x^{j}, t\right) d t \\
\forall \Psi \in H^{2,1}(Q),\left.\Psi\right|_{\Sigma}=0 \text { and } \Psi(x, T)=0 .
\end{gathered}
$$

Remark 1 The right hand side of (26) is a continuous linear form on $H^{2,1}(Q)$ if $n \leqslant 3$.

Consequently, after transformations, the first component on the left-hand side of (20) can be rewriten as

$$
\begin{gathered}
\lambda_{1} \sum_{j=1}^{\mu} \int_{0}^{T}\left(y\left(x^{j}, t ; v_{0}\right)-z_{d j}(t)\right)\left(y\left(x^{j}, t ; v\right)-y\left(x^{j}, t ; v_{0}\right)\right) d t= \\
=\int_{Q} p\left(v_{0}\right)\left(v-v_{0}\right) d x d t
\end{gathered}
$$

Substituting (27) into (20) we obtain

$$
\begin{array}{r}
\int_{Q}\left(p\left(v_{0}\right)+\lambda_{2} N v_{0}\right)\left(v-v_{0}\right) d x d t \geqslant 0 \\
\forall v \in U_{a d}
\end{array}
$$

Theorem 2 For the problem (1) - (4) with the cost function (17) with $z_{d j}(t) \in L^{2}(0, T)$ and $\lambda_{2}>0$ and with constraints on controls (18), there exists a unique optimal control $v_{0}$ which satisfies the maximum condition (28).

Consider now the particular case where $U_{a d}=L^{2}(Q)$. Thus the maximum condition (28) is satisfied when

$$
v_{0}=-\lambda_{2}^{-1} N^{-1} p\left(v_{0}\right)
$$

We must notice that the conditions of optimality derived above (Theorem 2) allow us to obtain an analytical formula for the optimal control in particular cases only (e.g. there are no constraints on controls). This results from the following: the determining of the function $p\left(v_{0}\right)$ in the maximum condition from the adjoint equation is possible if and only if we know $y_{0}$ which corresponds to the control $v_{0}$. These mutual connections make the practical use of the derived optimization formulas difficult. Therefore we resign from the exact determining of the optimal control and we use approximation methods. 
In the case of performance functional (17) with $\lambda_{1}>0$ and $\lambda_{2}=0$, the optimal control problem reduces to the minimizing of the functional on a closed and convex subset in a Hilbert space. Then, the optimization problem is equivalent to a quadratic programming one (Ref. [11]) which can be solved by the use of the well-known algorithms, e.g. Gilbert's (Ref. [11]).

The practical application of Gilbert's algorithm to an optimal control problem for a parabolic system with boundary condition involving a time delay is presented in [12]. Using Gilbert's algorithm, a one-dimensional numerical example of the plasma control process is solved.

\section{Conclusions}

The results presented in the paper can be treated as a generalization of the results concerning pointwise observation of the state given by the parabolic equation with the homogeneous Dirichlet boundary condition obtained in [13] onto the case of parabolic equations with the Neumann boundary conditions involving multiple constant time delays.

Sufficient conditions for the existence of a unique solution of such parabolic equations with the Neumann boundary conditions involving multiple constant time delays are proved (Lemma 1 and Theorem 1). The optimal control is characterized by using the adjoint equation (Lemma 2). The necessary and sufficient conditions of optimality are derived for a linear quadratic problem (1)-(4), (17), (18) (Theorem 2).

We can also obtain estimates and a sufficient condition for the boundedness of solutions for such parabolic time delay systems with specified forms of feedback control.

Finally, we can consider optimal control problems of time delay hyperbolic systems with pointwise observation of the state.

The ideas mentioned above will be developed in forthcoming papers.

\section{References}

[1] G. KNOWLES: Time-optimal control of parabolic systems with boundary conditions involving time delays. J. Optimiz. Theor. Applics., 25(4), (1978), 563-574.

[2] A. KowAlEwsKi: Optimal control with initial state not a priori given and boundary condition involving a delay. Lecture Notes in Control and Information Sciences, 95 (1987), 94-108, Springer-Verlag, Berlin-Heidelberg.

[3] A. KowAlewsKi: Boundary control of distributed parabolic system with boundary condition involving a time-varying lag. Int. J. Control, 48(6), (1988), 22332248 . 
[4] A. KowALEWSKI: Feedback control for a distributed parabolic system with boundary condition involving a time-varying lag. IMA J. Math. Control and Information, 7(2), (1990), 143-157.

[5] A. KowAlewsKi: Minimum time problem for a distributed parabolic system with boundary condition involving a time-varying lag. Arch. Automatic and Remote Control, XXXV(3-4), (1990), 145-153.

[6] A. KowALEWSKI: Optimality conditions for a parabolic time delay system. Lecture Notes in Control and Information Sciences, 144, (1990), 174-183, SpringerVerlag, Berlin-Heidelberg.

[7] A. KowalewsKi: Optimal control of parabolic systems with time-varying lags. IMA J. Math. Control and Information, 10( 2), (1993), 113-129.

[8] A. KowALEWSKI: Optimal control of distributed parabolic systems with multiple time time-varying lags. Int. J. Control, 69(3), (1998), 361-381.

[9] A. KowALEWSKI: Optimizaton of parabolic systems with deviating arguments. Int. J. Control, 72(11), (1999), 947-959.

[10] A. Kowalewski: Optimal control of time delay parabolic systems. Optimization, 50(1-2), (2001), 205-232.

[11] A. Kowalewski: Optimal control of infinite dimensional distributed parameter systems with delays. University of Mining and Metallurgy Press, Cracow, 2001.

[12] A. KowalewsKI and J. Duda: On some optimal control problem for a parabolic system with boundary condition involving a time-varying lag. IMA J. Math. Control and Information, 9(2), (1992), 131-146.

[13] J.L. LIONS: Optimal control of systems governed by partial differential equations. Springer-Verlag, Berlin-Heidelberg, 1971.

[14] J.L. Lions and E. MAGENES: Non-homogeneous boundary value problems and applications. 1 and 2, Springer-Verlag, Berlin-Heidelberg, 1972.

[15] P.K.C. WANG: Optimal control of parabolic systems wih boundary conditions involving time delays. SIAM J. Control, 13(2), (1975), 274-293.

[16] K.H. Wong: Optimal control computation for parabolic systems with boundary conditions involving time delays. J. Optimiz. Theor. Applics., 53(3), (1987), 475507. 